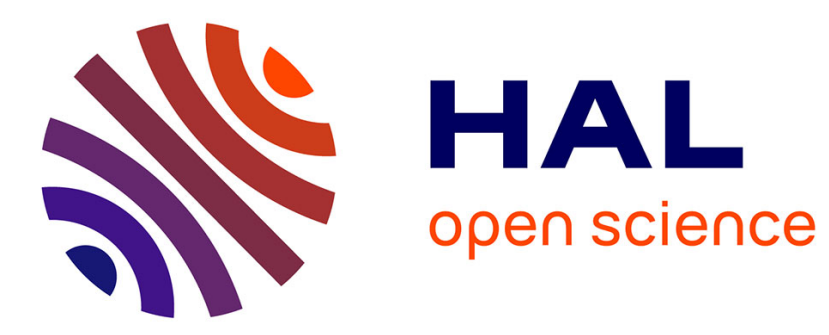

\title{
Vers des nouveaux types de réseaux sur Internet ? Les réseaux à liens faibles du dirigeant de petite entreprise
} Bernard Fallery, Carole Marti

\section{To cite this version:}

Bernard Fallery, Carole Marti. Vers des nouveaux types de réseaux sur Internet ? Les réseaux à liens faibles du dirigeant de petite entreprise. Revue management \& avenir, 2007, 12, pp.173-186. 10.3917/mav.013.0169 . hal-00775867

\section{HAL Id: hal-00775867 https://hal.science/hal-00775867}

Submitted on 14 Jan 2013

HAL is a multi-disciplinary open access archive for the deposit and dissemination of scientific research documents, whether they are published or not. The documents may come from teaching and research institutions in France or abroad, or from public or private research centers.
L'archive ouverte pluridisciplinaire HAL, est destinée au dépôt et à la diffusion de documents scientifiques de niveau recherche, publiés ou non, émanant des établissements d'enseignement et de recherche français ou étrangers, des laboratoires publics ou privés. 


\title{
Vers des nouveaux types de réseaux sur Internet ? Les réseaux à liens faibles du dirigeant de petite entreprise
}

\author{
Bernard FALLERY \\ Enseignant-chercheur à l'Université Montpellier 2, où il dirige le CREGO, laboratoire de \\ recherche de cette université. \\ bernard.fallery@univ-montp2.fr
}

\section{Carole MARTI}

Docteur de l'Université Montpellier 2, où elle dirige notamment un contrat de collaboration avec la Chambre Régionale de Métiers.

marti.carole@gmail.com

\author{
CREGO CC028 \\ Université Montpellier II, \\ Place E. Bataillon, \\ 34095 Montpellier
}

\section{Résumé :}

Nous présentons trois études de cas qui illustrent les nouveaux réseaux à la disposition du dirigeant de petite entreprise : importance des signaux faibles dans des clubs de dirigeants, utilisation d'Internet pour dé-problématiser la relation entre l'expert comptable et le dirigeant, partage et réutilisation de connaissances entre artisans via une base d'expériences «en ligne ». Aujourd'hui le dirigeant de PE peut disposer sur Internet de nouvelles sources informationnelles, pour construire un réseau relationnel innovant «à lien faible ».

\begin{abstract}
:
We present three case studies which illustrate the new networks at the disposal of the leader of small company: importance of the weak links in club of leaders, use of Internet for «dé-problématiser» the relation between the accountant and the leader, share and re-use of knowledge between craftsmen via a base of experiments "on line". Today the leader of small company can have on Internet news informational sources, to build a network relational innovating "with weak link".
\end{abstract}




\section{Introduction}

Comparativement aux grandes entreprises, les petites entreprises (PE) n'ont certes pas les mêmes moyens, en termes de temps ou de ressources financières, pour accéder à l'information. Aujourd'hui pourtant, le dirigeant de PE dispose lui aussi de sources informationnelles grâce aux TIC, et un des moyens les plus « évidents » pour disposer d'informations pertinentes est devenu de passer par ces nouveaux réseaux.

Dans les PE les réseaux sociaux ou institutionnels sont traditionnellement des réseaux personnels à liens forts (l'expert-comptable, le banquier, les fournisseurs...), mais certains réseaux plus purement informationnels (les stages de formation, les salons professionnels, l'accueil de stagiaires...) permettent la détection des indispensables signaux faibles. Comme dans une PE le rôle du dirigeant est déterminant sinon quasi exclusif dans ce processus, il est justement très important qu'il puisse s'approprier lui-même les outils qui lui permettront de mieux jouer son rôle d'interface : l'avantage concurrentiel peut alors venir, non pas de la créativité d'un seul individu, mais de la capacité à construire un réseau relationnel innovant «à liens faibles ». La capacité à innover semble en effet d'autant plus importante que les idées se diffusent via ces liens faibles (Ruef 2002) : les relations sociales entre des individus différents favorisent en effet l'adaptabilité à des situations nouvelles, puisque l'information sur de nouvelles opportunités y est plus facilement accessible. L'étude de Julien et al. (2002), concernant le comportement innovant des PE, montre d'ailleurs que les firmes les plus innovantes sont celles qui recourent le plus volontiers à ces liens faibles. La présence au sein d'un ensemble social d'une alternance de liens forts (réseaux dans lesquels les individus ont des relations régulières et fortes) et de liens faibles (réseaux dans lesquels les individus ont peu d'interactions) induit en effet les «trous structuraux » nécessaires à de nouvelles dynamiques sociales et à de nouvelles stratégies.

Quelles sont aujourd'hui les opportunités qu'offre Internet dans la construction de nouveaux réseaux à liens faibles pour l'information et le partage ? Pour répondre à ces questions nous feront le point dans une première partie sur ces concepts de liens forts et liens faibles à la suite de Granovetter (2000), Ruef (2002), Dibiaggio et Ferrary (2003), Burt (1992; 1995), et plus particulièrement dans le domaine de l'entrepreneuriat à la suite de Julien et al. (2002), Janissek-Muniz et Lesca (2003), Cochoy (2003).

Dans une seconde partie, nous illustrons ces opportunités par trois études de cas : des réseaux à liens faibles «en face-à-face » au sein de clubs de dirigeants, étude développée par P. Baillette $(2000 ; 2002)$, d'éventuels réseaux «par Internet » entre l'expert-comptable et la PE, étude menée par P. Chapellier $(1994 ; 2003 ; 2004)$, enfin des réseaux à liens faibles «sur Internet » entre des artisans qui peuvent aujourd'hui y partager leurs expériences, étude conduite par C. Marti (2005).

\section{Liens forts, liens faibles}

\subsection{Liens forts, liens faibles : un apport pour l'analyse des réseaux}

Granovetter $(1985$; 2000) définit les réseaux à liens faibles comme ceux avec lesquels les individus ont peu d'interactions dans le temps, une faible intensité émotionnelle, peu de confiance et peu de services réciproques. Les réseaux à liens faibles sont en fait constitués de sources moins fréquentées, à l'inverse de ceux à liens forts. 
Par le biais de l'analyse des réseaux sociaux, Granovetter (2000) montre que les interactions interindividuelles se traduisent par des phénomènes collectifs qui rétroagissent, de manière symétrique, sur les individus et les petits groupes. Au cœur de cette analyse se trouve la notion de «lien interpersonnel »:

" lien dont la force est une combinaison (probablement linaire) de la quantité de temps, de l'intensité émotionnelle, de l'intimité (la confiance mutuelle) et des services réciproques qui caractérisent ce lien »(Granovetter 2000, p. 46-47).

En fonction de l'intensité de la relation, le lien sera qualifié de fort, faible ou absent, et l'intensité des liens modifie la propension des agents à innover (Ruef 2002).

Les liens forts autour d'un individu forment un réseau dense, et les liens faibles un réseau lâche. Les liens forts sont sources de confiance et procurent une certaine sécurité, alors que les liens faibles donnent accès aux informations disponibles en dehors des cercles sociaux familiers (Granovetter 1985). Les liens forts sont des liens interactifs (Leonard-Barton and Sinha 1993) et seraient nécessaires pour l'échange de connaissances complexes, difficiles à codifier et souvent intégrées à un système de connaissances structurées (Hansen 1999). Dès 1973, Granovetter soutient un résultat contre intuitif, selon lequel l'efficacité informationnelle (la vitesse et la richesse de l'information diffusée dans l'ensemble d'un réseau) est en fait supérieure dans les réseaux à liens faibles car :

"les individus avec qui on est faiblement lié ont plus de chances d'évoluer dans des cercles différents, et ils ont donc accès à des informations différentes de celles que l'on reçoit $\gg$ (Granovetter 2000, p. 62).

Autrement dit, l'existence de liens faibles entre agents, c'est-à-dire de relations relativement peu fréquentes, est déterminante dans la dynamique de circulation de l'information dans les réseaux sociaux. L'absence de liens forts entre deux groupes constitue même ce que Burt (1992) appelle un « trou structurel ». Le «pont », c'est-à-dire alors le lien faible entre deux groupes autonomes, est fortement valorisable en ce qu'il est générateur de variété informationnelle dans chaque groupe. Plus ces trous structuraux et ces ponts dans un réseau sont nombreux, et plus les bénéfices informationnels du réseau seraient importants.

La création de liens faibles est loin d'être mécanique et elle est rarement le fruit du hasard. Elle repose souvent sur l'activation de ce que Dibiaggio et Ferrary (2003) appellent les « liens virtuels », au sens de liens «potentiels » entre différents groupes ou communautés. Les auteurs définissent ces liens comme des liens entre agents encastrés dans des structures sociales favorisant leur rencontre, tels les communautés ethniques, les associations culturelles ou sportives, ou les réseaux d'anciens étudiants ou de grandes entreprises. L'appartenance d'un individu à une association professionnelle lui confère ainsi des liens sociaux «potentiels ». En devenant membre d'une association un individu acquiert un capital social virtuel, et il a potentiellement accès à tous les membres de la communauté sans pour autant connaître chacun d'eux de manière personnelle. Il y a un lien potentiel entre tous les membres de la communauté, qui peut alors se concrétiser quand les acteurs le souhaitent (Ferrary and Pesqueux 2004). Pour Dibiaggio et Ferrary (2003), cette activation repose sur trois conditions:

(1) La présence de deux groupes indépendants, dont la relation est source de bénéfices potentiels ;

(2) L'existence d'un acteur tirant bénéfice de ce lien. La création d'un pont entre deux groupes autonomes est souvent l'œuvre d'un tiers qui reconnaît le bénéfice potentiel d'un trou structurel, et est donc incité à activer ce lien (Burt 1992). Ainsi, certains liens faibles, pourtant potentiellement valorisables, pourront rester inactivés si aucun acteur n'y trouve d'intérêt. 
(3) La troisième condition réside dans l'encastrement social des acteurs, rendant l'activation des liens non aléatoire. L'appartenance à un réseau social confère un capital social potentiel à ses membres, constitué par un ensemble de liens virtuels. Le fait d'appartenir à une communauté éthique, religieuse, d'être membre d'un réseau d'anciens, d'une grande école permet d'intégrer des communautés virtuelles et procure un potentiel de création de liens jusqu'alors inexistants. Ces liens virtuels peuvent être activés «en cas de nécessité ». Les institutions (universités, églises, entreprises, associations, partis politiques...) jouent un rôle essentiel dans la dynamique d'interactions sociales de création de liens faibles.

\subsection{Liens forts, liens faibles : un apport pour l'entrepreneuriat}

Cette problématique est prégnante dans le contexte de la PE. Comme le soulignent Julien et al. (2002), compte tenu de leurs ressources limitées, les PME doivent recourir à différents réseaux afin d'obtenir une information riche. Le rôle de ces réseaux est d'aider ces entreprises à profiter de sources informationnelles diverses. Les auteurs rappellent que dans les PME les réseaux sont essentiellement des réseaux personnels et d'affaires qui sont le plus souvent des réseaux forts (Clients, Fournisseurs...). Mais certains réseaux plus purement informationnels, qui peuvent comprendre aussi bien des réseaux sociaux qu'institutionnels, servent alors à compléter les informations provenant des réseaux personnels et d'affaires.

Comme dans une petite entreprise le rôle du dirigeant est déterminant sinon quasi exclusif dans le processus de veille, il est justement très important qu'il puisse s'approprier les outils qui lui permettront de mieux jouer son rôle d'interface : l'avantage concurrentiel peut alors venir, non pas uniquement de sa créativité personnelle, mais de sa capacité à construire un réseau relationnel innovant « à liens faibles » (Julien, Andriambeloson et al. 2002). Par opposition aux traditionnels réseaux à liens forts (que constitue par exemple l'expert-comptable, le banquier, les fournisseurs, les clients, la Chambre de Métiers... ) ces réseaux à liens faibles sont sociologiquement plus éloignés du dirigeant. Néanmoins, ils peuvent justement favoriser le repérage des signaux faibles que préconisent Janissek-Muniz et Lesca (2003), afin d'aider l'ensemble de l'entreprise à apprendre de façon permanente. De nombreux moyens «traditionnels » existent pour construire ces réseaux à liens faibles (les stages de formation, les clubs de dirigeants, les salons professionnels, la veille sur Internet, l'accueil de stagiaires, les expositions, le parrainage de seniors...).

Internet peut permettre aujourd'hui de décupler les capacités des petites entreprises, pour autant qu'elles sachent en profiter. Pour Granovetter (1973) ce sont bien les réseaux dans lesquels les acteurs sont encastrés qui vont déterminer les comportements individuels et le rendement de leurs échanges économiques, et Internet permettrait par exemple à presque toutes les petites entreprises de tisser des liens à l'international.

Cochoy (2003), dans un numéro de la revue «Réseaux » entièrement consacré aux TIC dans le cadre des petites entreprises, arrive à la conclusion suivante :

"Au travers des récits recueillis, on constate la réalisation de petites expériences et l'engagement d'apprentissages progressifs ; on observe chez les acteurs l'affirmation d'une remarquable capacité d'appropriation des outils et l'avènement d'une étonnante conscience réflexive de leurs potentialités (...) Internet permet aux petits acteurs, pour la première fois peut-être, d'être visibles sur le marché international sans devoir assumer des coûts de publicité hors de portée pour eux; Internet leur donne l'occasion de mobiliser gratuitement des informations sur le marché international sans avoir à se découvrir ; Internet leur fournit enfin les moyens de préserver d'anciennes relations et/ou de nouer de nouveaux contacts » (Cochoy 2003, p. 143). 
Ces nouveaux points de vue concernant la capacité d'innovation des petites entreprises sont dus en partie aux progrès réalisés dans son évaluation, et au fait que l'environnement économique et social a changé avec les évolutions technologiques, l'obsolescence et la mondialisation : nous serions dans une ère de «jeunes » entreprises (Jovanovic 2001). Mais un certain nombre d'explications théoriques ont aussi émergé pour expliquer les avantages des $\mathrm{PE}$ en matière d'innovation dans certains secteurs technologiques : l'absence de contraintes bureaucratiques, les nombreuses «petites » innovations trop modestes pour intéresser les grandes entreprises, les stratégies de saisie des opportunités, les innovations souvent commerciales et incrémentales, des proximités professionnelles et/ou communautaires, les externalités informationnelles au sein des réseaux, les systèmes productifs locaux, l'enthousiasme possible dans la petite entreprise où les liens entre les défis, le personnel et les récompenses potentielles sont beaucoup plus étroits.

\section{Trois études de cas : vers des nouveaux types de réseaux sur Internet}

Quelles sont les opportunités que peut offrir aujourd'hui Internet dans la construction de ces nouveaux réseaux à liens faibles pour l'information et le partage ? Pour répondre à cette question nous allons successivement présenter trois études de cas. La première a été développée par P. Baillette (2000; 2002), son objectif est de montrer en quoi l'appartenance à un réseau aide le dirigeant dans ses processus décisionnels. La deuxième a été menée par $\mathrm{P}$. Chapellier $(1994 ; 2003$; 2004) sur la relation assez problématique entre l'expert-comptable et le dirigeant de PE. Enfin la troisième étude a été menée au sein d'une Chambre Régionale des Métiers et de l'Artisanat (Marti 2005) et montre comment une base d'expériences facilite le partage et la réutilisation de connaissances entre artisans via une base de données en ligne.

Dans le tableau ci-dessous nous reprenons les trois études avec leurs caractéristiques principales, leurs principaux objectifs et la méthodologie employée par le chercheur.

Tableau 1 : Récapitulatif des trois études de cas

\begin{tabular}{|l|l|l|l|}
\hline & Baillette (2000; 2002) & $\begin{array}{l}\text { Chapellier (1994; 2003; } \\
\text { 2004) }\end{array}$ & Marti (2005) \\
\hline $\begin{array}{l}\text { Population } \\
\text { étudiée }\end{array}$ & $\begin{array}{l}\text { Propriétaires-dirigeants } \\
\text { de petites entreprises }\end{array}$ & $\begin{array}{l}\text { Expert-comptable \& } \\
\text { dirigeants de petites } \\
\text { entreprises }\end{array}$ & Artisans ${ }^{2}$ \\
\hline $\begin{array}{l}\text { Type de } \\
\text { réseaux }\end{array}$ & $\begin{array}{l}\text { Association de } \\
\text { dirigeants }\end{array}$ & $\begin{array}{l}\text { Relation entre l'expert- } \\
\text { comptable \& le dirigeant } \\
\text { PE }\end{array}$ & $\begin{array}{l}\text { Chambres de Métiers avec } \\
\text { un outil de gestion des } \\
\text { connaissances en ligne }\end{array}$ \\
\hline $\begin{array}{l}\text { Objectifs du } \\
\text { réseau }\end{array}$ & $\begin{array}{l}\text { Aide au processus } \\
\text { décisionnel du } \\
\text { dirigeant }\end{array}$ & $\begin{array}{l}\text { Déproblématiser leurs } \\
\text { relations }\end{array}$ & $\begin{array}{l}\text { Partage et réutilisation des } \\
\text { connaissances }\end{array}$ \\
\hline Méthodologie & $\begin{array}{l}\text { Étude de cas sur } 3 \\
\text { associations }-39 \\
\text { entretiens }\end{array}$ & $\begin{array}{l}\text { Revue de la littérature } \\
(2003 ; 2004) \\
\text { Enquête sur 113 dirigeants }\end{array}$ & Recherche action sur 48 cas \\
\hline
\end{tabular}

\footnotetext{
${ }^{1}$ Petites entreprises de moins de cinquante salariés.

${ }^{2}$ Un artisan est un entrepreneur qui investit sur son savoir faire. Son statut juridique est défini par un ensemble de contraintes. Il doit exercer une activité professionnelle de production, de transformation, de réparation ou de prestation de service relevant de l'artisanat. Il doit être économiquement indépendant et être immatriculé au répertoire des métiers. Et enfin, il ne peut pas employer plus de dix personnes lors de sa création.
} 


\begin{tabular}{|l|l|l|l|}
\hline & & de PE & \\
\hline Résultats & $\begin{array}{l}\text { Le réseau aide répondre } \\
\text { aux interrogations des } \\
\text { décideurs }\end{array}$ & $\begin{array}{l}\text { Les experts-Comptables } \\
\text { sont freinés par l'utilisation } \\
\text { d'Internet }\end{array}$ & $\begin{array}{l}\text { L'accès une base } \\
\text { d'expériences favorise le } \\
\text { partage et la réutilisation }\end{array}$ \\
\hline
\end{tabular}

\subsection{Le cas des Clubs de dirigeants : les liens faibles, en face-à-face}

Cette recherche conduite par P. Baillette (2000; 2002) s'intéresse au système d'information du dirigeant d'entreprise, et plus particulièrement celui constitué par ses contacts et par les associations censées lui apporter une aide dans ces activités professionnelles. En effet, tant les chefs d'entreprises que la communauté scientifique soulignent l'intérêt des activités relationnelles développées à l'intérieur de tels réseaux pour faciliter l'accès des entreprises à des sources informationnelles diverses. Plus la taille de l'entreprise va être petite et plus ces activités vont même constituer un intérêt stratégique au vu de leurs ressources restreintes. Les « associations» ou les «clubs » fréquentés par les dirigeants recouvrent des catégories très diversifiées de réseaux, l'étude se concentre sur les «associations de dirigeants à finalité de réflexion et d'action sur la gestion de l'entreprise ». Ce type d'association réunit des individus ayant la même activité de dirigeant et les mêmes responsabilités, au sein de réunions régulières et formalisées. L'étude porte sur une population de propriétaires dirigeants de petites entreprises (moins de cinquante salariés) adhérant à ce type de clubs. L'auteur examine trois associations : le centre des jeunes dirigeants (CJD), l'association Rhodanim, et le groupement des chefs d'entreprise du Québec. La finalité de ces associations est de contribuer au développement des entreprises adhérentes et de leur apporter un soutien dans leurs activités.

Dans ces clubs l'intérêt des dirigeants de PE se porte sur la création et le développement de leurs réseaux sociaux informationnels et sur la valorisation de ce capital social (Baillette 2002). En effet les «classiques » liens forts constitués au sein du réseau restreint du dirigeant n'offrent pas la richesse informationnelle de ces liens faibles, tissés notamment avec des collègues, des amis, des voisins, mais développés ici grâce à l'appartenance à une association de dirigeants. Les liens faibles vont alors permettre de construire des «ponts» avec un plus grand nombre d'individus, qui, grâce à leur appartenances multiples, ont accès à davantage d'informations et peuvent faciliter les transmissions de signaux faibles. Alors que ces relations développées en dehors de l'entreprise sont parfois considérées par les dirigeants comme une activité secondaire, elles fournissent au contraire ici l'occasion d'échanger de l'information «potentiellement» utile. L'étude de Baillette $(2000 ; 2002)$ se consacre à l'ensemble du processus décisionnel du dirigeant. Le modèle décisionnel utilisé comprend les quatre phases classiques d'intelligence (reconnaissance et compréhension de stimuli), de modélisation (développement d'une ou plusieurs solutions au problème posé), de choix (sélection parmi les solutions possibles), et enfin de mise en œuvre de la solution choisie. En considérant l'ensemble de ce processus, on montre bien que l'information ne sert pas seulement à prendre des décisions (c'est-à-dire à opérer des choix, problem solving) mais elle est également utilisée dans toutes les autres étapes du processus. En particulier, l'information peut-être exploitée dans les phases amont du processus décisionnel pour interpréter et clarifier les faits, recueillir des informations pour mieux connaître l'environnement et pouvoir anticiper (problem finding). Le dirigeant à la tête 
d'une PE est le véritable «centre nerveux » de l'entreprise. Cette position lui donne accès à pratiquement toutes les sources informationnelles dans l'entreprise. En comparaison des grandes entreprises, le dirigeant de PE doit faire face à des conditions spéciales caractérisées en particulier par une pénurie relative de ressources, surtout en ce qui concerne les moyens financiers et humains. Dès lors, les dirigeants de PE sont amenés à développer eux-mêmes des contacts avec l'environnement. Cette activité relationnelle apparaît donc particulièrement importante pour les dirigeants de PE, car elle leur permet de bénéficier d'informations privilégiées en mettant en œuvre des moyens limités (Duchéneaut 1996; Julien 1996).

À partir de ces constats, Baillette $(2000 ; 2002)$ pose la question suivante : Les réseaux de type «clubs de dirigeants » peuvent-ils être un outil pertinent pour l'aide à la décision?

Dans ces associations ou clubs, les adhérents rencontrent des homologues exerçant la même activité de dirigeants d'entreprise et les réunions au sein de ces structures sont généralement formalisées (agenda, ordre du jour, animation...). L'auteur définit le « succès » de l'aide à la décision apportée par ce type d'association en référence aux trois critères classiques : l'efficacité, l'efficience et la satisfaction. L'aide est alors perçue par l'adhérent comme étant efficace si l'association l'a aidé à atteindre les buts qu'il s'était fixé. L'aide est perçue comme étant efficiente s'il a réalisé des économies de moyens (utiliser moins de ressources qu'il n'en aurait consommé avec d'autres sources, ou bien améliorer les résultats obtenus pour un même investissement).

Il ressort de l'étude (1) que l'intérêt des chefs d'entreprise est très lié à leur mission «théorique », et (2) que le succès de l'aide apporté par l'association est grandement renforcé par l'assiduité des membres :

"Les contacts noués grâce au réseau permettent en effet d'économiser des moyens grâce à un accès facilité aux acteurs adéquats. L'économie réalisée se manifeste particulièrement en termes de temps, d'énergie et d'argent. Le succès perçu de l'aide, le climat de convivialité qui existe dans ce type de réseaux, contribuent à la satisfaction des dirigeants membres dans l'évaluation de l'aide apportée »(Baillette 2002, p. 118).

Au final, ce type de réseau de liens faibles en face-à-face remplit de manière satisfaisante son objectif, c'est-à-dire être un moyen capable d'offrir une aide à la formulation et à l'interprétation des problèmes, idées et questions que se pose le dirigeant dans son rôle de décideur.

\subsection{Le cas des relations dirigeants et experts-comptables : les liens par Internet}

La seconde étude de cas a été menée par Chapellier $(2003 ; 2004)$ pour étudier la relation entre l'expert-comptable et le dirigeant de PE. Ici la littérature présente en général une vision réductrice de ces relations (orientées principalement vers la production des documents obligatoires, dans le but de satisfaire aux obligations imposées par l'administration fiscale), alors que pour prendre leurs décisions les dirigeants de petites entreprises auraient essentiellement recours à leurs jugements, intuitions et expériences (Fallery 2001).

Selon Chapellier $(2003 ; 2004)$ la faible utilisation de l'information comptable dans ce type d'entreprise est liée au fait que le dirigeant n'a pas toutes les clés pour tirer profit de cette information, bien que l'environnement dans lequel il se trouve (souvent considéré par luimême comme " quasi-désertique ») induirait la recherche d'un compagnonnage avec une personne qui puisse lui apporter des conseils. La majorité des PE ne disposent pas de service 
comptable interne capable de produire et d'interpréter les données, et ce serait donc à l'expert-comptable que reviendrait normalement la mission de production, d'analyse et de diffusion de cette information. Mais les experts-comptables, bien que conscients de ce besoin, ne parviennent pas toujours à le satisfaire. Plusieurs études démontrent en effet que la mission de l'expert-comptable dans les PE reste très éloignée des attentes des dirigeants. Ces besoins pas toujours satisfaits perturbent la relation entre l'expert-comptable et son client.

Dans ce contexte, les TI pourraient jouer un rôle de support important dans le processus de diffusion des connaissances. De nombreuses études soulignent que l'usage d'Internet, et plus particulièrement de la messagerie électronique, n'est pas une entrave à une communication riche et variée. Ce média est efficace si les différents acteurs respectent les règles du jeu, s'ils engagent par exemple à aller chercher régulièrement les messages dans leurs boîtes aux lettres et à répondre de manière instantanée (Chanal 2000). Ce qui importe, c'est le comportement social de l'individu et la disponibilité allouée à son utilisation (Guilloux, Gauzente et al. 2000). L'usage de la messagerie électronique pourrait donc faciliter la relation entre l'expert-comptable et son client : le dirigeant pose sa question à l'expertcomptable, celui-ci, à des moments qui lui conviennent, consulte sa messagerie, fait les recherches utiles, et rédige la réponse. Si l'expert-comptable répond dans un délai raisonnable, le dirigeant aura moins la sensation d'un manque de disponibilité et d'accessibilité liée à la difficulté de le rencontrer face-à-face ou de le joindre au téléphone. Si l'Intranet du cabinet est connecté à celui du client, celui-ci pourra accéder aux informations comptables à toutes heures. Il pourra suivre l'avancement de son dossier et réagir à tout moment si nécessaire. Comme la messagerie offre la possibilité de transmettre des documents attachés, cette fonction pourrait accroître la qualité de service grâce par exemple à la transmission de bulletins de salaires en pièces jointes, qui permettrait au client de visualiser instantanément le résultat du travail effectué au cabinet et d'apporter si nécessaire des corrections ou des instructions complémentaires. L'adressage multiple permet enfin une large diffusion mais aussi la transparence de l'information.

Globalement, Chapellier (2003; 2004) montre que les apports potentiels de l'Internet pour la mission de l'expert-comptable dans les PE sont multiples. Il favorise le partage de l'information et facilite la communication. Il devrait permettre de mieux travailler ensemble en complétant les liens forts «classiques ». Internet devrait permettre une réduction du temps consacré dans les cabinets à l'accomplissement de tâches techniques, qu'il s'agisse de formalités administratives ou de saisie comptable. De plus en plus d'administrations nationales ou locales offrent la possibilité d'accomplir les démarches par Internet. La profession comptable s'est dotée d'un portail télé-déclaratif « jedeclare.com », conduisant à une dématérialisation généralisée des déclarations, et le Conseil Supérieur de l'Ordre a par ailleurs signé avec plusieurs banques un protocole d'accord national destiné à organiser le téléchargement et la saisie automatique des données bancaires des clients. Les experts comptables se verront ainsi libérés des tâches à faible valeur ajoutée, et disposeront de plus de temps pour mieux répondre aux attentes de leurs clients. L'usage d'Internet peut en ce sens être porteur d'une nouvelle logique de fonctionnement des cabinets susceptible de « déproblématiser » la relation entre l'expert-comptable et son client. Il existe cependant de nombreux freins, d'un point de vue technique (car les informations échangées entre l'expertcomptable et le dirigeant de PE sont en partie considérées comme sensibles), mais aussi organisationnel (si certains individus y voient un progrès important dont ils vont profiter, d'autres y voient un risque de devoir changer des habitudes et de perdre un certain pouvoir). 
Pourtant le Conseil de l'Ordre des Experts-comptables essaie de faire évoluer les pratiques et de jouer un rôle sur deux points essentiels : d'une part convaincre les expertscomptables de l'enjeu technologique (le thème est très largement abordé dans les revues, documentations, congrès de la profession), et d'autre part accompagner et former afin de faciliter la phase d'appropriation de la technologie. De nombreuses formations sont régulièrement organisées sur des thèmes aussi divers que la sécurité des échanges dématérialisés, les anti-virus et pare-feux, la comptabilité, la paye, et la gestion en applications à distance, l'organisation des téléprocédures et des télétransmissions, la création de sites web ...

Des informations à jour, visualisables en temps réel, un expert-comptable plus disponible, plus accessible : L'étude de Chapellier montre que ces améliorations pourraient réduire l'intensité des critiques actuellement formulées par les dirigeants de PE à l'encontre de leur expert-comptable, et déclencher ainsi une dynamique positive avec plus de conseils et plus de confiance.

\subsection{Le cas du partage entre artisans : le développement de liens faibles par Internet}

Cette troisième étude a consisté à mettre en place un outil de gestion des connaissances sur Internet pour une Chambre Régionale des Métiers et de l'Artisanat (CRMA). Le rôle d'une CRMA consiste à réaliser des études prospectives, afin d'orienter le développement à moyen terme du secteur de l'artisanat et du fonctionnement des Chambres régionales. C'est ainsi que les TIC constituent un axe de travail en plein essor au sein des Chambres de Métiers, et la CRMA a souhaité mener un projet d'action en faveur du développement de la compétitivité des artisans. Elle justifie ce choix par l'analyse du contexte, et part de l'idée que l'utilisation de ces technologies est un facteur de développement et de gains de compétitivité, sous réserve que cette utilisation soit maîtrisée et cohérente avec le métier de l'entreprise : le secteur est particulièrement réceptif à l'innovation, du fait du pragmatisme des artisans, de leur culture technique et de la capacité d'adaptation des petites entreprises. L'optimisme de ce constat ne doit, cependant, pas masquer le risque d'une fracture technologique qui exclurait une partie des artisans des évolutions en cours, faute de temps, de moyens ou de compétences adaptées.

L'outil spécialement construit pour cette étude devait répondre à ce besoin latent : il regroupe « en ligne » un certain nombre d'histoires racontées par des artisans à propos de leurs usages des TIC. Grâce à cet outil les artisans vont pouvoir tisser des réseaux à liens faibles afin d'acquérir des connaissances sur l'utilisation des TIC par d'autres artisans. On a pu alors montrer qu'une base de récits d'expériences peut favoriser un réseau à liens faibles permettant d'assurer l'apprentissage, grâce aux possibilités de recueil, de partage et de réutilisation.

Une fois l'outil mis en place on a pu ensuite observer comment il a été utilisé par les artisans. L'objectif opérationnel de cet outil est que les artisans puissent lire en ligne les expériences d'autres artisans (phase de partage), pour se forger une idée sur des usages possibles d'Internet et qu'éventuellement ils s'en servent pour leur propre activité (phase de réutilisation). Un des objectifs de l'étude était alors de voir comment il pouvait y avoir réutilisation de connaissances en accédant aux expériences des autres. On peut donc parler ici d'une création de liens faibles par une activation d'un lien virtuel entre deux groupes qui tirent profit de cette relation. Pour reprendre les termes de Granovetter (2000), cette relation n'est pas aléatoire, puisque l'appartenance des artisans aux réseaux des Chambres de Métiers 
leur confèrent potentiellement un «capital social », la CRMA joue un rôle essentiel dans la dynamique d'interactions sociales de création de liens faibles.

L'artisan désireux de partager des connaissances dispose déjà de différents moyens, que ce soient des réseaux à liens forts (fournisseurs, clients...) ou des réseaux à liens faibles (stages, expositions, syndicats professionnels..). Aujourd'hui, l'outil de gestion de connaissances mis en place sur Internet fournit une nouvelle source de connaissances complémentaire : le protocole d'expérimentation a permis non seulement de vérifier qu'il avait bien création de liens faibles entre des artisans qui ne se rencontrent pas en face à face, mais qu'ils peuvent profiter des connaissances développées par d'autres. L'étude s'est déroulée sur quarante-huit cas d'artisans, et on a pu constater que tous ne réutilisaient pas de la même façon : certains vont plutôt envisager de réutiliser des histoires proches de leur contexte professionnel, alors que d'autres au contraire vont pouvoir réutiliser des histoires éloignées de leur sphère d'activité. On a montré ici que l'activation de liens faibles par Internet afin de partager et de réutiliser des connaissances peut s'avérer fructueuse.

\section{Conclusion}

À travers ces trois études de cas, différentes illustrations des réseaux à la disposition du dirigeant de PE ont été présentées. L'étude de P. Baillette (2002) montre l'importance des signaux faibles «en face-à-face » dans des clubs de dirigeants pour améliorer notamment la phase «d'intelligence » dans le processus de décision. L'étude de Chapellier (2003) montre les difficultés inhérentes à la relation de l'expert-comptable et du dirigeant de petites entreprises, et conclut à une utilisation possible d'Internet pour « dé-problématiser » cette relation. Enfin dans la dernière étude de cas Marti (2005) montre comment le développement de liens faibles grâce à Internet permet le partage et la réutilisation de connaissances, puisque l'artisan peut disposer aujourd'hui d'un espace pour l'échange d'expériences.

\section{Bibliographie :}

Baillette, P. (2000). L'importance des activités relationnelles pour l'aide à la décision. Le cas de l'adhésion du propriétaire dirigeant de PME à une association de dirigeants. Sciences de Gestion. Montpellier, Université de Montpellier 2.

Baillette, P. (2002). "L'aide à la décision par l'adhésion à une association de dirigeants à finalité de réflexion et d'action sur la gestion de l'entreprise." Systèmes d'Information et Management 7(2): 105-128.

Burt, S. R. (1992). Structural holes : the social structure of competition. Cambridge, Harvard University Press.

Burt, S. R. (1995). "Le capital social, les trous structuraux et l'entrepreneur." Revue Française de Sociologie XXXVI(4): 599-628.

Chanal, V. (2000). La structuration d'un projet d'innovation par la communication électronique. Congrès de l'AIMS, Montpellier.

Chapellier, P. (1994). Comptabilités et système d'information du dirigeant de PME : essai d'observation et d'interprétation des pratiques. Montpellier, Université de Montpellier 2: 368. 
Chapellier, P. (2003). "Les apports potentiels de l'Internet à la mission de l'expert-comptable dans les petites entreprises." Revue Comptabilité Contôle Audit 9(2): 171-187.

Chapellier, P. (2004). Internet pour une relation de qualité entre l'expert-comptable et le dirigeant de PE. Congrès de l'Association Fracophone de Comptabilité, Orléans.

Cochoy, F. (2003). "La "toile" comme force des points faibles?" Réseaux 121: 119-144.

Dibiaggio, L. et M. Ferrary (2003). "Communautés de pratique et réseaux sociaux dans la dynamique de fonctionnement des clusters de hautes technologies." Revue d'économie industrielle(103): 111-130.

Fallery, B. (2001). "Nouvelles technologies et travail du dirigeant." Systèmes d'Information et Management (SIM) 6(2): 81-91.

Ferrary, M. et Y. Pesqueux (2004). L'organisation en réseau, mythes et réalités. Paris, Presse Universitaire de France.

Granovetter, M. (1973). "The strength of weak ties." American Journal of Sociology 78(6): 1360-1380.

Granovetter, M. (1985). "Economic action and social culture : the problem of embeddedness." American Journal of Sociology 78(6): 481-510.

Granovetter, M. (2000). Le marché autrement : les réseaux dans l'économie. Paris, Desclée de Brouwer.

Guilloux, V., C. Gauzente, et al. (2000). Grandeurs et limites de la communication électronique : analyse d'un cas de projet en recherche marketing. Congès de l'AFM, Montréal.

Hansen, M. T. (1999). "The search-transfer problem : the role of weak ties in sharing knowledge across organization subunits." Administrative Science Quarterly 44(1): 82-111.

Janissek-Muniz, R. et H. Lesca (2003). Veille stratégique : application d'internet et sites web pour « provoquer » des informations à caractère anticipatif. Association Information et Management (AIM), Grenoble.

Jovanovic, B. (2001). "New technology and the small firm." Small Business Economics 16(1): 53-55.

Julien, P. A., E. Andriambeloson, et al. (2002). Réseaux, signaux faibles et innovation technologique dans les PME du secteur des équipements de transport terrestre. 6ème congrès international francophone de la PME, HEC Montréal.

Leonard-Barton, D. et D. K. Sinha (1993). "Developer-user interaction and user satisfaction in internal technology transfer." Academy of Management Journal 36(5): 1125-1139.

Marti, C. (2005). L'apport des méthodes narratives à la gestion des connaissances : le partage et la réutilisation entre artisans. Sciences de Gestion. Montpellier, Université Montpellier 2: 398. 
Ruef, M. (2002). "Strong ties, weak ties and islands : structural and cultural predictors of organizational " Industrial and Corporate Change 11(3): 427-449.

Bernard FALLERY est enseignant-chercheur à l'Université Montpellier 2, où il dirige le CREGO, laboratoire de recherche de cette université.

Carole MARTI est docteur de l'Université Montpellier 2, où elle dirige notamment un contrat de collaboration avec la Chambre Régionale de Métiers.

Adresse Institutionnelle

CREGO CC028 - Université Montpellier II， Place E. Bataillon, 34095 Montpellier

Mail fallery@polytech.univ-montp2.fr marti@iae.univ-montp2.fr 